This item was submitted to Loughborough's Research Repository by the author.

Items in Figshare are protected by copyright, with all rights reserved, unless otherwise indicated.

\title{
Generation of rayleigh-type waves on plate edges by laser-initiated airborne shock waves
}

PLEASE CITE THE PUBLISHED VERSION

http://dx.doi.org/10.3813/AAA.918343

\section{PUBLISHER}

(c) S. Hirzel Verlag on behalf of the European Acoustics Association

\section{VERSION}

AM (Accepted Manuscript)

\section{LICENCE}

CC BY-NC-ND 4.0

\section{REPOSITORY RECORD}

Georgiev, Vasil B., Victor V. Krylov, Qin Qin, and Keith Attenborough. 2012. "Generation of Rayleigh-type Waves on Plate Edges by Laser-initiated Airborne Shock Waves". figshare. https://hdl.handle.net/2134/10998. 
This item was submitted to Loughborough's Institutional Repository (https://dspace.lboro.ac.uk/) by the author and is made available under the following Creative Commons Licence conditions.

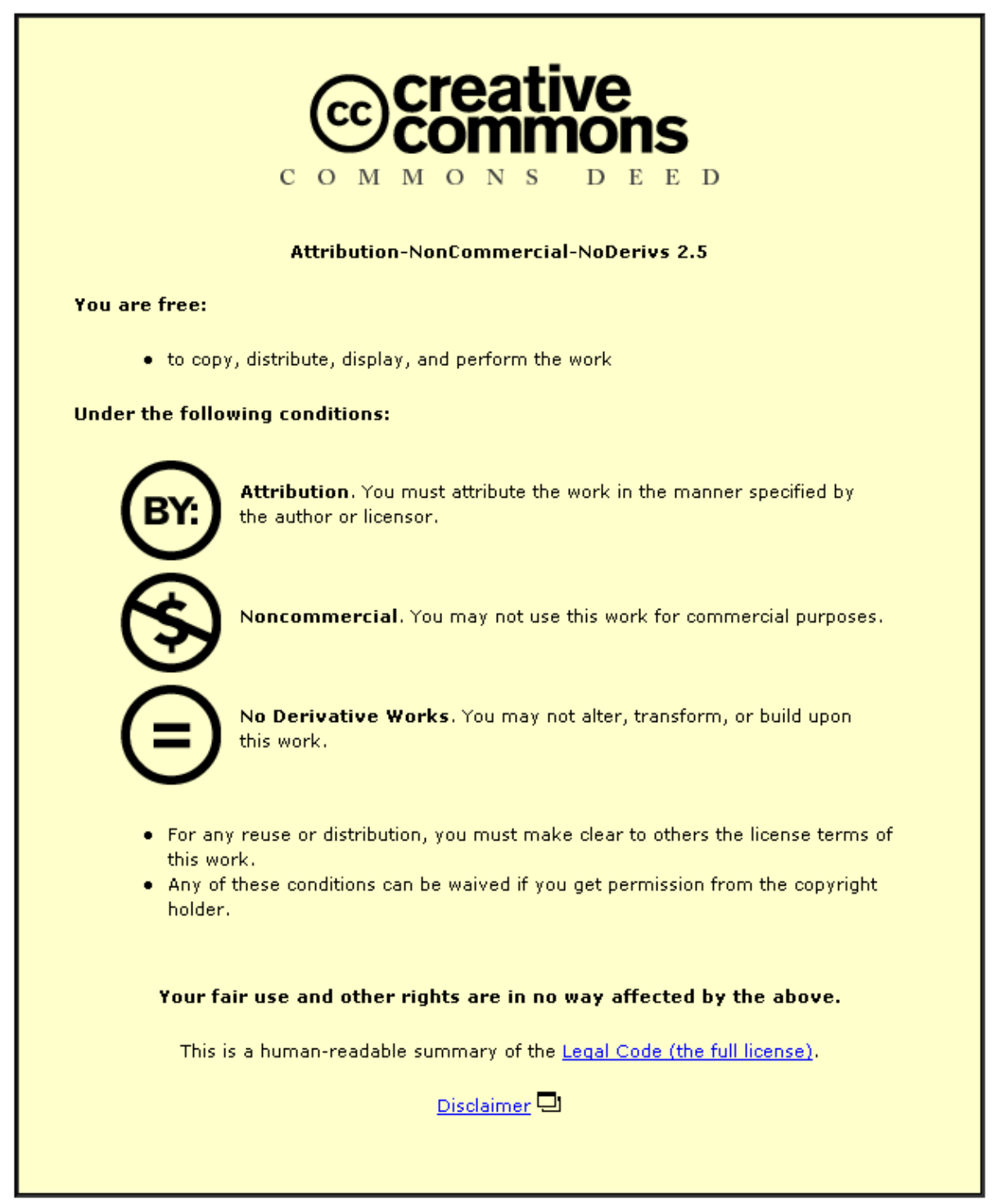

For the full text of this licence, please go to: http://creativecommons.org/licenses/by-nc-nd/2.5/ 


\section{Generation of Rayleigh-type Waves on Plate Edges}

\section{by Laser-initiated Airborne Shock Waves}

Vasil B. Georgiev ${ }^{\text {a }}$, Victor V. Krylov ${ }^{\text {a,* }}$, Qin Qin ${ }^{\text {b }}$, Keith Attenborough ${ }^{\text {c }}$,

${ }^{a}$ Department of Aeronautical and Automotive Engineering, Loughborough University, Ashby Road, Loughborough, Leicestershire, LE11 3TU, UK;

b Acoustic Research Centre, Department of Engineering, University of Hull, Cottingham Road, Hull, HU6 7RX, UK;

${ }^{\mathrm{c}}$ Department of Engineering, University of Hull, Cottingham Road, Hull, HU6 7RX, UK.

\section{Summary}

The present paper describes the results of the semi-analytical modelling of the interaction of laser-initiated air shock waves with the edge of an infinite vertically mounted elastic plate. The impact of the shock wave on the plate edge is approximated by an equivalent

\footnotetext{
* Corresponding author. Tel.: +44 1509 227216; E-mail address: V.V.Krylov@lboro.ac.uk
} 
edge force resulting from the combined pressure of the incident and reflected shock waves. This force is then represented in the wavenumber-frequency domain by means of Fourier transforms that are carried out numerically. After that the problem is solved using the Green's function method. The resulting frequency spectra and time histories of generated Rayleigh-type wave pulses propagating along the plate edge are calculated for different heights of the laser beam focusing above the plate edge. The obtained theoretical results are compared with the results of the laboratory experiments on laser-initiated air shock wave interaction with an edge of a large vertically mounted Perspex plate that is used for reduced-scale modelling of blast wave interaction with the ground surface. The resonant properties of the accelerometer have been taken into account to describe the received signals. The comparison shows that the obtained semi-analytical results are in good agreement with the experiments.

PACS numbers: 43.28.Mw, 43.40.Dx, 43.40.Jc, 43.40.Qi

\section{Introduction}

During military tests, various types of controlled explosions take place above the ground surface. Such explosions generate not only shock waves in air but also strong ground vibrations that may annoy local residents [1,2]. Studying these effects using full-scale field experiments is costly and time consuming. It is more convenient and much less expensive to study the associated sound and vibration phenomena using reduced-scale laboratory measurements, with a laser as a source of airborne shock waves [3] interacting 
with large horizontally positioned elastic plates modelling the ground [4]. In spite of a number of useful modelling properties of horizontal plate configurations, flexural waves excited in such plates by shock waves have a specific velocity dispersion that is rather different from the dispersion of Rayleigh surface waves in the ground. Strictly speaking, waves induced in horizontal plates are more appropriate for modelling elastic waves generated in the ice of a frozen lake rather than in the ground. But to increase the thickness of a plate to model an elastic half space, which might be a better representation of real ground, is impractical because of a very large mass of such a structure. Consequently a more realisable laboratory model of a homogeneous elastic ground is a vertically mounted thin elastic plate, so that the generated plate waves have in-plane displacements.

The aim of the present paper is to investigate Rayleigh-type edge waves generated due to the interaction of laser-initiated airborne shock waves with an edge of a vertically positioned large thin elastic plate providing the so-called plane stress condition (see Fig. 1). It is well known that such a plate can support essentially non-dispersive Rayleigh-type waves propagating along a free edge of a plate. The role of shear waves in this case is played by the so-called shear-horizontal $(\mathrm{SH})$ plate mode propagating with a speed of shear waves in the plate material, $c_{t}$, whereas the role of longitudinal waves is played by the quasi-longitudinal (symmetric) plate mode propagating with the so-called plate velocity, $c_{p}=c_{l}\left(1-c_{t}^{2} / c_{l}^{2}\right)^{1 / 2}$, where $c_{l}$ is the speed of longitudinal waves in the plate material.

Many investigations of laser-generated Rayleigh surface waves in solids have been carried out in the past (see for example [5-9]). However, in all these investigations a laser beam was interacting directly with a solid surface, and Rayleigh waves, as well as bulk longitudinal and shear waves were generated by means of the so-called thermo-optical generation mechanism. This mechanism is associated with the conversion of the incident 
optical energy into heat at the solid surface and subsequent thermal expansion of the heated area. Due to a relatively small size of the heated area of the resulting thermooptical sources, the entire physical picture in this situation is very similar to the wellknown case of wave generation in elastic half-space by mechanical forces applied to a limited sub-surface area. However, this picture is not adequate for model experiments on laser-initiated airborne shock wave interaction with an edge of a vertical plate, where a laser beam is focused in the air outside a plate edge. The resulting laser-initiated airborneshock wave interacting with a plate edge represents a complex and essentially distributed source varying both in space and time. Moreover, for a shock wave interaction with an edge of a thin plate, the initially three-dimensional geometry of a spherically diverging air shock wave is transformed into the two-dimensional geometry of generated Rayleigh-type edge waves and quasi-longitudinal and SH waves.

A strong shock wave in the air as a distributed source for elastic wave generation in solids has been investigated earlier by one of the present authors in respect of generation of Rayleigh surface waves by electric spark discharge near the surface [10]. In that investigation, a semi-analytical method to describe interaction of air shock waves with an elastic half space was developed. More recently, the generation of flexural waves in thin plates due to laser-induced airborne shock waves has been studied by the present authors both theoretically and experimentally [11,12].

The aim of the present paper is to further develop the above-mentioned semianalytical approach [10] and to apply it to generation of Rayleigh-type edge waves in plates by laser-initiated airborne shock waves. The impact of the incident air shock waves is approximated by an equivalent edge force resulting from the combined surface pressure of the incident and reflected shock waves. The well-known analytical expressions for shock wave velocity and pressure in the front of a shock wave are used to describe this 
surface force as a function of time and distance from the epicentre. The problem is then solved using the integral solution for a two-dimensional half-space. The resulting frequency spectra and time histories of the generated Rayleigh-type wave pulses are calculated for different values of height of the laser-generated spark above the plate edge. The predicted time histories and frequency spectra of generated Rayleigh-type edge waves are compared with data from laboratory experiments involving laser-initiated airborne shock wave interaction with an edge of a vertical Perspex plate.

\section{Theoretical background}

\subsection{Specification of the equivalent edge force}

The wave velocity $v^{\text {sh }}(r)$ and pressure $P^{s h}(r)$ in the front of a spherically diverging airborne shock wave can be described by the following analytical expressions [13]:

$$
\begin{aligned}
& v^{s h}(r)=\frac{2}{5} \zeta_{0}^{5 / 2}\left(\frac{E}{\rho_{0}}\right)^{1 / 2} r^{-3 / 2}, \\
& P^{s h}(r)=\left(\frac{2}{5}\right)^{2} \frac{2 \zeta_{0}^{5}}{\gamma+1} E r^{-3} .
\end{aligned}
$$

Here $E$ is the energy instantly released in the origin of a spherically diverging shock wave, $\rho_{0}$ is the mass density of the air, $\gamma \approx 1.41$ is the Poisson adiabatic constant, and $\zeta_{0}$ 
$\approx 0.93$ is the dimensionless parameter characterizing the self-similar motion of the shock wave front [13]. The superscript "sh" stands for shock waves.

During the interaction between an airborne shock wave with the edge of a vertical plate, a considerable part of the shock wave energy incident upon the edge is reflected back into the air. But the remainder is transformed into the energy of Rayleigh-type edge waves and into the energy of SH and quasi-longitudinal plate waves propagating in the bulk of the plate.

To evaluate the air pressure acting on the plate edge, the latter can be approximated as absolutely rigid. In this case, the edge pressure can be considered as the sum of the incident and reflected pressures. Using the usual linear acoustic reflection coefficient for a rigid surface (equal to 1), the resulting edge pressure can be expressed simply as twice the pressure in the incident wave [10].

Using the expressions (1) and (2) valid for the shock wave front, the entire pressure pulse due to the air shock wave will be approximated by the two exponential functions [11,12]. This reflects the fact that for medium and large distances, which are of main interest for the experiments to be described below, the shock wave pressure initially undergoes a discontinuous jump above the atmospheric pressure. Then it decreases below the atmospheric pressure, and finally returns to its initial value.

The air pressure (in respect of the atmospheric pressure) can then be expressed as follows (see also Fig. 2): 


$$
P^{s h}(r, t)=P^{s h}(r)\left\{\begin{array}{l}
H\left(t-t_{0}^{s h}\right)\left[1.2 \exp \left(-\left(t-t_{0}^{s h}\right) \frac{1}{\varepsilon t_{0}^{s h}}\right)-0.2\right]- \\
H\left(t-t_{0}^{s h} 3.5\right)\left[1.2 \exp \left(-\left(t-t_{0}^{s h}\right) \frac{0.36}{\varepsilon t_{0}^{s h}}\right)-0.2\right]
\end{array}\right\} .
$$

Here $H(t)$ is the Heaviside step function, $\varepsilon=0.05$ is a constant controlling the duration of the pressure pulse, and

$$
t_{0}^{s h}=\left(\frac{r}{\zeta_{0}}\right)^{5 / 2}\left(\frac{\rho_{0}}{E}\right)^{1 / 2}
$$

In Eqn (4), the distance $r$ from the origin of laser-induced acoustic shock to the point of observation on the surface is equal to $\sqrt{\rho^{2}+h^{2}}$ (see Fig. 1(upper)).

Using the above-mentioned expression for air pressure (Eqn (3)) and the linear acoustic approximation for the reflection coefficient, the equivalent force normal to the surface can be expressed as:

$$
F_{n}^{s h}(r, t)=2 P^{s h}(r, t),
$$

Using Fourier transform, the frequency spectrum of the above function can be expressed as follows: 


$$
F_{n}^{s h}(\omega, \rho)=\frac{P^{s h}(r)}{\pi}\left\{\begin{array}{l}
\frac{1.2 \varepsilon t_{0}^{s h}}{1-i \omega \varepsilon t_{0}^{s h}} \exp \left(i \omega t_{0}^{s h}\right)-\frac{0.2}{i \omega}\left[\exp \left(i \omega t_{0}^{s h} 3.5\right)-\exp \left(i \omega t_{0}^{s h}\right)\right]- \\
\frac{1.2 \varepsilon t_{0}^{s h}}{0.36-i \omega \varepsilon t_{0}^{s h}} \exp \left(-\frac{0.9}{\varepsilon}+i \omega t_{0}^{s h} 3.5\right)
\end{array}\right\} .
$$

Note that the above expressions for the equivalent surface force (Eqns (3) and (6)) have been derived using Eqns (1) and (2) for velocity and pressure in a strong air shock wave [13]. However, for distances $r$ that are large enough, say $r>r_{0}$, where $r_{0}$ is the distance at which the particle velocity at a shock front becomes equal to the speed of sound, the above-mentioned expressions for surface forces are no longer valid. Figure 3 shows how particle velocity at the shock wave front changes with the distance from the origin.

In this case the air particle velocity and pressure beyond the distance $r_{0}=0.0084 \mathrm{~m}$ (defined from Eqn (1) for $v_{0}=340 \mathrm{~m} / \mathrm{s}$ and $E=0.8 \mathrm{~J}$ (the laser energy per pulse)), have to be calculated using the characteristics of a normal acoustic wave propagating with the speed of sound $v_{0}$. The wave pressure in this case is inversely proportional to the distance: $P(r)=A / r$, where the constant $A$ should be determined by equalizing the air pressure in the shock wave to the acoustic wave pressure at $r=r_{0}$. Moreover, it should be remembered that the time duration of a pressure pulse in a strong shock wave increases proportionally to the distance from the epicenter, whereas in a normal acoustic wave this duration is constant. Thus the time duration of the pressure pulses at distance $r=r_{0}$ should be equalized as well.

For an acoustic wave, Eqns (2) and (3) should be replaced by the following expressions: 


$$
\begin{aligned}
& v^{a c}=v_{0}=340 \mathrm{~m} / \mathrm{s} \\
& P^{a c}(r)=\left(\frac{2}{5}\right)^{2} \frac{2 \zeta_{0}^{5}}{\gamma+1} \frac{E}{r_{0}^{2} r},
\end{aligned}
$$

where the superscript “ $a c$ ” stands for acoustic waves.

Using the above equations, one can obtain the following expression for pressure valid at $r>r_{0}$ :

$$
P^{a c}(r, t)=P^{a c}(r)\left\{\begin{array}{l}
H\left(t-t_{0}^{a c}\right)\left[1.2 \exp \left(-\left(t-t_{0}^{a c}\right) \frac{1}{\varepsilon t_{0}^{s h}\left(r_{0}\right)}\right)-0.2\right]- \\
H\left(t-t_{0}^{a c}-2.5 t_{0}^{s h}\left(r_{0}\right)\right)\left[1.2 \exp \left(-\left(t-t_{0}^{a c}\right) \frac{0.36}{\varepsilon t_{0}^{s h}\left(r_{0}\right)}\right)-0.2\right]
\end{array}\right\},
$$

where $t_{0}^{a c}=t_{0}^{s h}\left(r_{0}\right)+\frac{r-r_{0}}{v_{0}}$.

Using again the linear acoustics approximation for the reflection coefficient, the equivalent normal force per unit area acting on the plate edge can be considered to be the doubled incident pressure, and the frequency spectrum of the equivalent normal force can be expressed as: 


$$
F_{n}^{a c}(\omega, \rho)=\frac{P^{a c}(r)}{\pi}\left\{\begin{array}{l}
\frac{1.2 \varepsilon t_{0}^{s h}\left(r_{0}\right)}{1-i \omega \varepsilon t_{0}^{s h}\left(r_{0}\right)} \exp \left(i \omega t_{0}^{a c}\right)-\frac{0.2}{i \omega}\left[\exp \left(i \omega\left(t_{0}^{a c}+t_{0}^{s h}\left(r_{0}\right)\right)\right)-\exp \left(i \omega t_{0}^{a c}\right)\right]- \\
\frac{1.2 \varepsilon t_{0}^{s h}\left(r_{0}\right)}{0.36-i \omega \varepsilon t_{0}^{s h}\left(r_{0}\right)} \exp \left(-\frac{0.9}{\varepsilon}+i \omega\left(t_{0}^{a c}+2.5 t_{0}^{s h}\left(r_{0}\right)\right)\right)
\end{array}\right\}
$$

\subsection{Rayleigh-type wave generation on the plate edge}

In this section a two-dimensional problem of Rayleigh-type wave excitation on a plate edge is considered. The equivalent surface force applied to the unit element of area has been specified in the previous section. In the case under consideration this equivalent surface force should be multiplied by the plate thickness $d$ in order to determine the equivalent edge force acting on a unit length of the plate edge. After that, this problem does not differ from the well-known problem of elastic wave excitation in a 2D half-space (plain strain condition) by a line force applied in the normal direction to the surface (along the $\mathrm{x}$ axis) in XZ plane (in this case the coordinate $\rho$ is replaced by $x$ ).

In the case of 2D half-space excitation, the components of the elastic displacements $u$ and $w$ in rectangular coordinate system can be written down using the displacement potentials $\varphi$ and $\psi$ as [14]

$$
\begin{aligned}
& u=\frac{\partial \varphi}{\partial x}-\frac{\partial \psi}{\partial z} \\
& w=\frac{\partial \varphi}{\partial z}+\frac{\partial \psi}{\partial x}
\end{aligned}
$$


The boundary conditions in this case can be expressed as follows:

$$
\begin{aligned}
& {\left[p_{z x}\right]_{z=0}=\mu\left(2 \frac{\partial^{2} \varphi}{\partial x \partial z}+\frac{\partial^{2} \psi}{\partial x^{2}}-\frac{\partial^{2} \psi}{\partial z^{2}}\right)=0} \\
& {\left[p_{z z}\right]_{z=0}=\lambda \nabla^{2} \varphi+2 \mu\left(\frac{\partial^{2} \varphi}{\partial z^{2}}+\frac{\partial^{2} \psi}{\partial x \partial z}\right)=F_{n}(\omega, x)}
\end{aligned},
$$

where,

$$
F_{n}(\omega, x)=\left\{\begin{array}{ll}
F_{n}^{s h} d, & 0<x<x_{0} \\
F_{n}^{a c} d, & x>x_{0}
\end{array}\right\}
$$

Now the solution for the components of displacements, $u_{0}$ in $x$ direction and $w_{0}$ in $z$ direction, on the surface of the equivalent 2D half-space can be expressed as follows [14]:

$$
\begin{aligned}
& u_{0}=\frac{i}{\mu} \int_{-\infty}^{\infty} \frac{k\left(2 k^{2}-k_{t}^{2}-2 v_{l} v_{t}\right)}{F(k)} F_{n}(\omega, k) e^{-i k x} d k \\
& w_{0}=-\frac{1}{\mu} \int_{-\infty}^{\infty} \frac{k_{t}^{2} v}{F(k)} F_{n}(\omega, k) e^{-i k x} d k
\end{aligned}
$$

where $v_{l, t}^{2}=k^{2}-k_{l, t}^{2}$, and $k_{l, t}=\omega / c_{l, t}$ are the wavenumbers of longitudinal and shear bulk acoustic waves, $c_{l}=\left[(\lambda+2 \mu) / \rho_{p l}\right]^{1 / 2}$ and $c_{t}=\left(\mu / \rho_{p l}\right)^{1 / 2}$ are their phase velocities, $F(k)=\left(2 k^{2}-k_{t}^{2}\right)^{2}-4 k^{2} v_{l} v_{t}$ is the Rayleigh determinant. There is only one real zero of 
$F(k)$ that represents the wavenumber of the Rayleigh wave, $k_{R}=\omega / c_{R}$, where $c_{R}$ is Rayleigh wave velocity.

For the case of a plate edge under consideration one should replace $c_{l}$ by $c_{p}$, and $c_{R}$ by $c_{R T}$, where $c_{R T}$ is the velocity of Rayleigh-type waves on a plate edge, whereas $c_{t}$ remains unchanged.

Finally,

$$
F_{n}(\omega, k)=\frac{1}{\pi} \int_{0}^{x_{0}} F_{n}^{s h}(\omega, x) d e^{i k x} d x+\int_{x_{0}}^{\infty} F_{n}^{a c}(\omega, x) d e^{i k x} d x
$$

is the Fourier transform of the equivalent edge force from the frequency-distance to the frequency-wavenumber domain.

The spectral components of the displacements of generated Rayleigh waves can be evaluated from Eq. (13) using the method of residues:

$$
\begin{aligned}
& u_{0}=-\frac{2 \pi}{\mu} \frac{k_{R}\left(2 k_{R}^{2}-k_{\beta}^{2}-2 v v^{\prime}\right)}{F^{\prime}\left(k_{R}\right)} F_{n}\left(\omega, k_{R}\right) e^{-i k_{R} x} \\
& w_{0}=-\frac{2 \pi i}{\mu} \frac{k_{\beta}^{2} v}{F^{\prime}\left(k_{R}\right)} F_{n}\left(\omega, k_{R}\right) e^{-i k_{R} x}
\end{aligned}
$$

where $F^{\prime}\left(k_{R}\right)$ is the first derivative of Rayleigh determinant over $k$ taken at $k=k_{R}$.

The above components of displacements have been calculated for the residue at $k=k_{R}$. These expressions, combined with the harmonic time factor, $e^{-i \omega t}$, represent a Rayleigh-type edge wave propagating from the epicenter of the acoustic shock. 
The vertical components of the particle velocity and acceleration can be obtained from the vertical displacement $w_{0}$ (see Eqn. (15)) as:

$$
\dot{w}_{0}(\omega, x)=-i \omega w_{0}(\omega, x) \quad \text { and } \quad \ddot{w}_{0}(\omega, x)=-\omega^{2} w_{0}(\omega, x)
$$

The time history of the vertical displacement pulse can be calculated from Eqn (15) using the inverse Fourier transform:

$$
w_{0}(t, \rho)=\int_{-\infty}^{\infty} w_{0}(\omega, \rho) e^{-i \omega t} d \omega
$$

\section{Experimental studies and their comparison with the theory}

A Perspex plate with the dimensions 1400 x 25 x $600 \mathrm{~mm}$, i.e. with the value of thickness $d=0.025 \mathrm{~m}$, was mounted vertically in a semi-anechoic chamber. The laser used to generate the sparks was a Q-switch Surelite III-10 Nd: YAG laser with a $1064 \mathrm{~nm}$ wavelength and energy of $0.8 \mathrm{~J}$ per pulse $[3,4]$. The gas breakdown induced by the laser has a duration of between 4 and 6 nanoseconds. For this duration of the pulse the pulse power is between 133 and $200 \mathrm{MW}$. A convex lens with focal length of $10 \mathrm{~cm}$ has been used to focus the beam to a spot of diameter of about $0.3 \mathrm{~mm}$ so that the light intensity in the focused spot is between $1.88 \times 10^{11}$ and $2.83 \times 10^{11} \mathrm{~W} / \mathrm{cm}^{2}$. At the distance of $3 \mathrm{~cm}$ from the spark, the peak pressure of the laser-generated air shock wave measured by a microphone was $181 \mathrm{~dB}$ re $20 \mu \mathrm{Pa}(22683 \mathrm{~Pa})$. The frequency spectrum of this shock wave lies between $3 \mathrm{kHz}$ and $159 \mathrm{kHz}$, with a peak at $20 \mathrm{kHz}$ [4]. The analysis system 
used in the experiments consisted of a conditioning amplifier, B\&K Type 2636; an NI 5911 data acquisition card enabling sampling rates as high as 100 million samples per second and LabView software.

For measurements of vibrations associated with generated Rayleigh-type waves propagating along the plate edge, an accelerometer with resonant frequency of $9.2 \mathrm{kHz}$ was used. Measurements were carried out for different distances from the epicenter: 100, 150, 200, 250, 300, 350 and $400 \mathrm{~mm}$. The height of laser generated sparks over the plate edge was $3 \mathrm{~mm}$. Further details regarding the laboratory equipment and test rig can be found in the papers $[3,4]$.

In what follows, some results of the above-mentioned semi-analytical approach are compared with data from the experimental measurements. The material characteristics (Acrylonitrile Butadiene Styrene, or ABS) of the vertical plate used in the numerical calculations are: mass density $\rho_{s}=1070 \mathrm{~kg} / \mathrm{m}^{3}$, Lame's parameters $\lambda=3.15 \cdot 10^{9} \mathrm{~N} / \mathrm{m}^{2}$, $\mu=0.9 \cdot 10^{9} \mathrm{~N} / \mathrm{m}^{2}$, and speed of sound in the air was taken as $c=340 \mathrm{~m} / \mathrm{s}$. Using the above parameters, the phase velocities of longitudinal and shear bulk waves could be calculated respectively as follows: $c_{l}=2150.9 \mathrm{~m} / \mathrm{s}$ and $c_{t}=917.1 \mathrm{~m} / \mathrm{s}$.

The results shown in Fig. 4 represent the predicted amplitude spectra and time histories of the Rayleigh-type edge wave vibration velocity at the distance of $0.3 \mathrm{~m}$ away from the epicenter. The assumed heights of laser-generated sparks above the plate edge are 2.5, 3.0 and $3.5 \mathrm{~mm}$. As it can be seen, increasing the height of the laser-generated spark leads to a decrease in the values of the amplitude spectra, pulse elongation and reduction in peak-to-peak amplitudes of the pulse waveforms. In addition, the spectral components are shifted towards lower frequencies. 
Figures 5 and 6 (lower) show the time histories of acceleration pulses measured at distances 250, 300 and $200 \mathrm{~mm}$ from the epicenter in the laboratory experiments. As was mentioned earlier, the height of the spark was $3 \mathrm{~mm}$ from the plate edge. After allowing for the difference in the durations that are plotted, it can be seen that the data differ from the theoretical calculations by the presence of decaying oscillations. Based on the frequency spectrum (Figure 6 (upper)) corresponding to the measured acceleration pulse at the distance of $200 \mathrm{~mm}$ (see Fig. 6), these oscillations are attributable to the strong peak at about $9.2 \mathrm{kHz}$. This peak occurs for all measurements, and is apparently related to the resonant frequency of the accelerometer.

Note that peak amplitudes of all three acceleration pulses shown in Figures 5 and 6 are roughly the same for all distances from the epicenter. This provides indirect experimental support for the fact that the observed pulses represent Rayleigh-type edge waves. Indeed, as it is well known, these are the only waves in this configuration that are one-dimensional (propagating along the edge) and, thus, are not attenuated due to geometrical spreading. Moreover the material attenuation is rather low at these frequencies.

Figure 7 shows experimental time-delayed acceleration pulses for the first few oscillations. For clarity, only three pulses are shown - for the distances of 100, 200 and 300 $\mathrm{mm}$ from the epicenter. Dividing the changes in distances by the corresponding time delays, one can estimate the phase velocity of propagating wave pulses as $1170 \mathrm{~m} / \mathrm{s}$. This value is somewhat higher than the calculated value of shear wave velocity $c_{t}=917.1 \mathrm{~m} / \mathrm{s}$ (see above), although for Rayleigh-type waves it should be lower. This discrepancy could be explained by a possible deviation of the material parameters of the real experimental plate from the parameters of Acrylonitrile Butadiene Styrene (ABS) used in the above calculations. In spite of this discrepancy, the measured value of velocity, $1170 \mathrm{~m} / \mathrm{s}$, is still 
much closer to the calculated shear wave velocity $c_{t}=917.1 \mathrm{~m} / \mathrm{s}$ than to the calculated longitudinal wave velocity $c_{l}=2150.9 \mathrm{~m} / \mathrm{s}$. This gives further support, as well as the above-mentioned equal amplitudes, for the fact that the observed acceleration pulses are pulses of Rayleigh-type edge waves.

The response of an accelerometer to a Rayleigh-type wave pulse can be calculated by multiplying its frequency response function (FRF) and the amplitude spectrum of the generated Rayleigh-type pulse given by Eqn (15). Taking an inverse Fourier transform from this product, one can obtain the calculated time history of the acceleration measured by an accelerometer. The FRF of a typical accelerometer can be expressed as follows [15]:

$$
H_{a}(\omega)=\left(\frac{i T \omega}{1+i T \omega}\right)\left(\frac{1}{\omega_{0}^{2}-\omega^{2}+2 \varsigma \omega}\right),
$$

where $T=R C$ is the accelerometer's time constant (in this case $T=0.5 \mathrm{~s}$ was used), $\omega_{0}$ is the resonant frequency of the accelerometer (assumed to be $9.2 \mathrm{kHz}$ ) and $\varsigma=1 \%$ of $\omega_{0}$ is the accelerometer's damping ratio. The accelerometer's FRF calculated according to Eqn (18) is shown in Fig. 8.

The amplitude spectra of the calculated accelerometer responses at the epicentral distance of $200 \mathrm{~mm}$ produced by a source at $3 \mathrm{~mm}$ and $6 \mathrm{~mm}$ are presented in Fig, 9. Figure 10 shows the calculated time histories of the acceleration pulses for a source at 3 $\mathrm{mm}$ and $6 \mathrm{~mm}$. Now, the calculated acceleration time histories can be directly compared to the experimental data shown in Fig. 6.

It can be seen that, similarly to the experimental results, the calculated acceleration pulses represent decaying oscillations at the resonant frequency $(9.2 \mathrm{kHz})$. The small discrepancy between the data and predictions could be due to the different numbers of 
resolution points used. In spite of this small discrepancy, the time histories of the acceleration pulses predicted after allowing for the accelerometer FRF are in reasonably good agreement with the experimental data.

Essentially, the short pulses of shock-induced Rayleigh-type waves excite the accelerometer at its resonant frequency, which generates corresponding electric signals with narrow spectra around this frequency associated with the observed decaying oscillations. As a result of the relatively low resonant frequency of the accelerometer used in the experiments, it was not possible to observe the complete spectra of generated Rayleigh-type waves. Nevertheless, the agreement of other observed parameters with the predictions demonstrates that the reported semi-analytical theory can be used successfully to describe the experiments on generation of Rayleigh-type edge waves in plates by laserinitiated air shock waves.

\section{Conclusions}

In the present paper, a semi-analytical model of Rayleigh-type wave generation on the edge of a thin vertical plate due to airborne shock waves has been developed. As might be expected, it is predicted that generated Rayleigh-type wave pulses depend strongly on the height of the laser spark from the surface: the lower the height the larger the amplitudes of the pulses.

Laboratory data, obtained using laser-initiated airborne shock waves incident on a vertical perspex sheet, at different distances from the epicenter of the shock wave, exhibit decaying oscillations at the resonant frequency of the accelerometer. Nevertheless the observed amplitudes and times of arrival are consistent with the generation of shock- 
induced Rayleigh waves. Moreover, after allowing for the frequency response of the accelerometer, the data are in reasonable accord with the predictions of the developed semi-analytical approach.

\section{Acknowledgments}

The research reported here has been supported by EPSRC grant EP/E027121/1. 


\section{References}

[1] K. Attenborough, P. Schomer, F. v.d. Eerden, E. Vedy, Overview of the theoretical development and experimental validation of blast sound-absorbing surfaces, Noise Control Engineering Journal 53(3) (2006) 70 - 80.

[2] P. Schomer, K. Attenborough, Basic results from full-scale tests at Ft Drum, Noise Control Engineering Journal 53(3) (2006) 94 - 109.

[3] K. Attenborough and Q. Qin, Model experiments on shock wave interaction with the ground, Proceedings of the $19^{\text {th }}$ International Congress of Acoustics, Madrid, Spain, 27 September 2007 (on CD).

[4] Q. Qin and K. Attenborough, Characteristics and application of laser-generated acoustic shock waves in air, Applied Acoustics 65 (2004) 325-340.

[5] R. White, Generation of elastic waves by transient surface heating, Journal of Applied Physics 34(12) (1963) 3559-3567

[6] C. Scruby, R. Dewhurst, D. Hutchins, S. Palmer, Quantitative studies of thermallygenerated elastic waves in laser irradiated metals, Journal of Applied Physics 51 (1980) 6210-6216. 
[7] V.V. Krylov, V.I. Pavlov, Thermooptical excitation of surface acoustic waves in solids, Soviet Phys. - Acoust., 28(6) (1982) 493-494.

[8] P. Doyle, C. Scala, Near-field ultrasonic Rayleigh waves from a laser line source, Ultrasonics 34 (1996) 1-8.

[9] J. Bernstein, J. Spicer, Line source representation for laser-generated ultrasound in aluminum, Journal of the Acoustical Society of America 107(3) (2000) 1352-1357.

[10] V.V. Krylov, On the theory of surface acoustic wave generation by electric spark discharge, J. Phys. D: Appl. Phys. 25 (1992) 155-161.

[11] V.B. Georgiev, V.V. Krylov, Q. Qin, K. Attenborough, Generation of flexural waves in infinite plates by laser-initiated air shock waves, Proceedings of the Institute of Acoustics 30, Pt. 2 (2008) 147-154.

[12] V.B. Georgiev, V.V. Krylov, Q. Qin, K. Attenborough, Semi-analytical modelling of plate flexural waves generated by laser initiated air shock waves, Proceedings of the International Conference “Acoustics 08”, Paris, France, June 2008, pp 1883-1888 (on CD).

[13] Y.B. Zel'dovich, Y.P. Raizer, Physics of shock waves and high temperature hydrodynamic phenomena, Vols. 1 and 2, New York, Academic Press (1966, 1967). 
[14] W.M. Ewing, W.S. Jardetzky, F. Press, Elastic waves in layered media, New York, McGraw-Hill (1957).

[15] K.G. McConnell, Vibrating testing: theory and practice, New York, John Wiley \& Sons (1995). 


\section{Figure captions}

Figure 1: Interaction of a laser-initiated airborne shock wave with an edge of a vertical plate: geometry of the problem (above), view of the experimental rig (below)

Figure 2: Calculated air pressure pulses in a shock wave calculated according to Eqn (3)

Figure 3: Calculated shock front velocity as a function of height $h$ of the laser spark and distance $\rho$ from the epicenter - (above); calculated pressure pulse in an acoustic wave according to Eqn (8) - (below)

Figure 4: Calculated amplitude spectra (above) and time histories (below) of the Rayleigh-type wave velocity pulses for different heights of the laser-generated spark

Figure 5: Measured time histories of the acceleration pulses of Rayleigh-type waves at distances of $250 \mathrm{~mm}$ (above) and $300 \mathrm{~mm}$ (below) from the epicenter

Figure 6: Measured amplitude spectrum (above) and time history (below) of the acceleration pulse of generated Rayleigh-type wave at the distance of $200 \mathrm{~mm}$ from the epicenter 
Figure 7: Measured time-delayed acceleration pulses (first oscillations) at the distance of 100, 200 and $300 \mathrm{~mm}$ from the epicenter

Figure 8: Calculated transfer function $\left|\mathrm{H}_{\mathrm{a}}\right|$ of an accelerometer having a resonant frequency at $9.2 \mathrm{kHz}$ according to Eqn (18)

Figure 9: Calculated acceleration amplitude spectrum of a generated Rayleigh-type wave after allowing for the calculated accelerometer FRF; the distance is $200 \mathrm{~mm}$ from the epicenter, and heights are $0.003 \mathrm{~m}$ (solid curve) and $0.006 \mathrm{~m}$ (dotted curve)

Figure 10: Calculated time histories of the acceleration pulses of generated Rayleigh-type waves at the distance of $200 \mathrm{~mm}$ from the epicenter predicted after allowing for the calculated accelerometer FRF; heights are $0.003 \mathrm{~m}$ (above) and 0.006 m (below) 

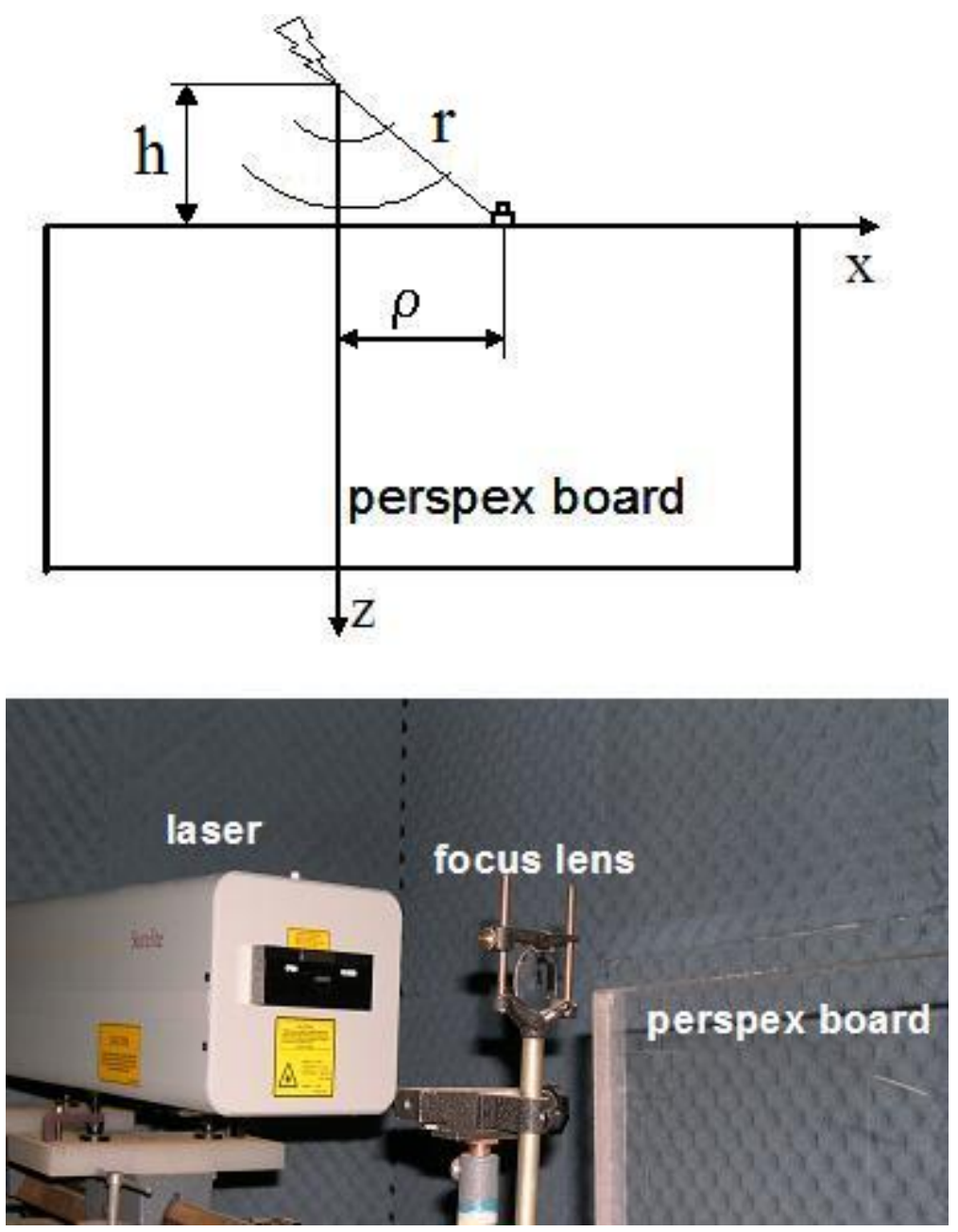

Figure 1: Interaction of a laser-initiated airborne shock wave with an edge of a vertical plate: geometry of the problem (above), view of the experimental rig (below) 


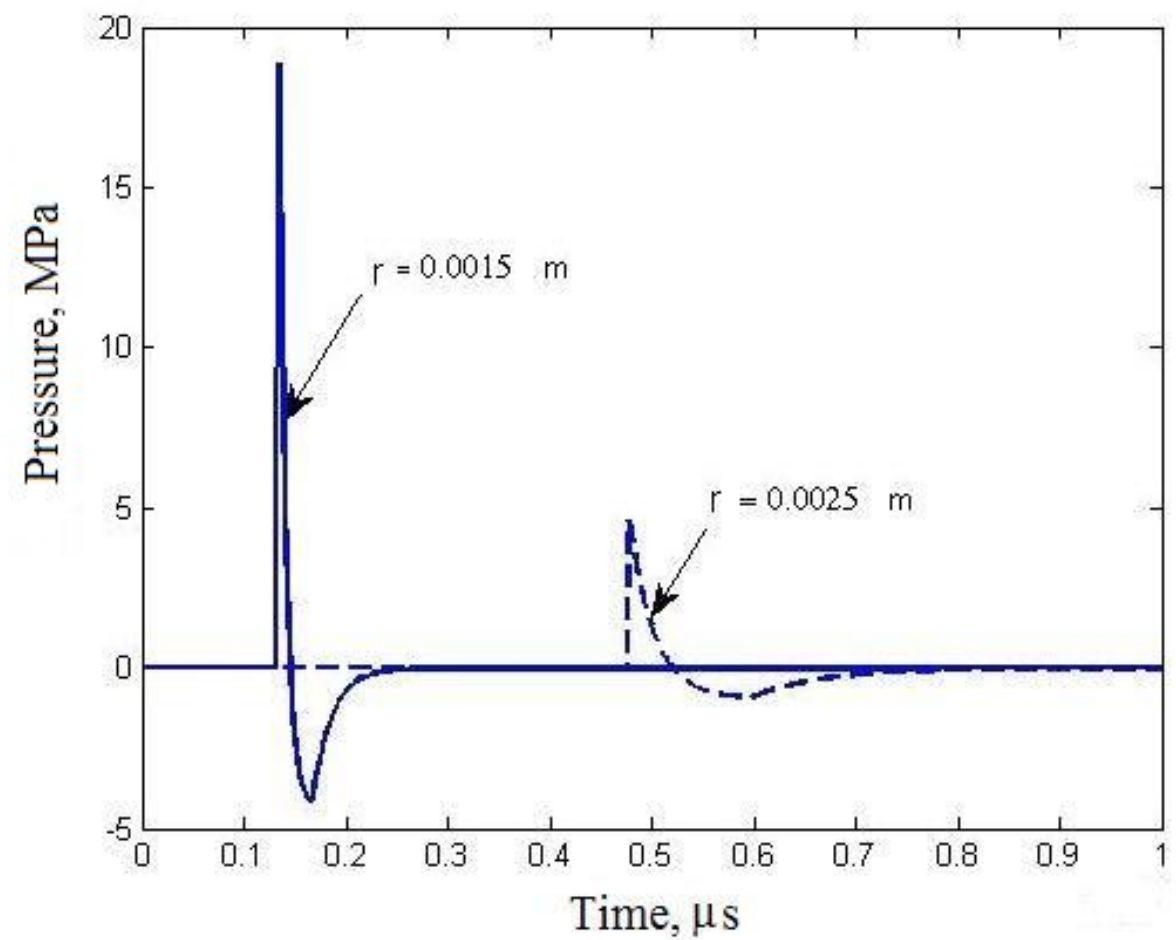

Figure 2: Calculated air pressure pulses in a shock wave according to Eqn (3) 


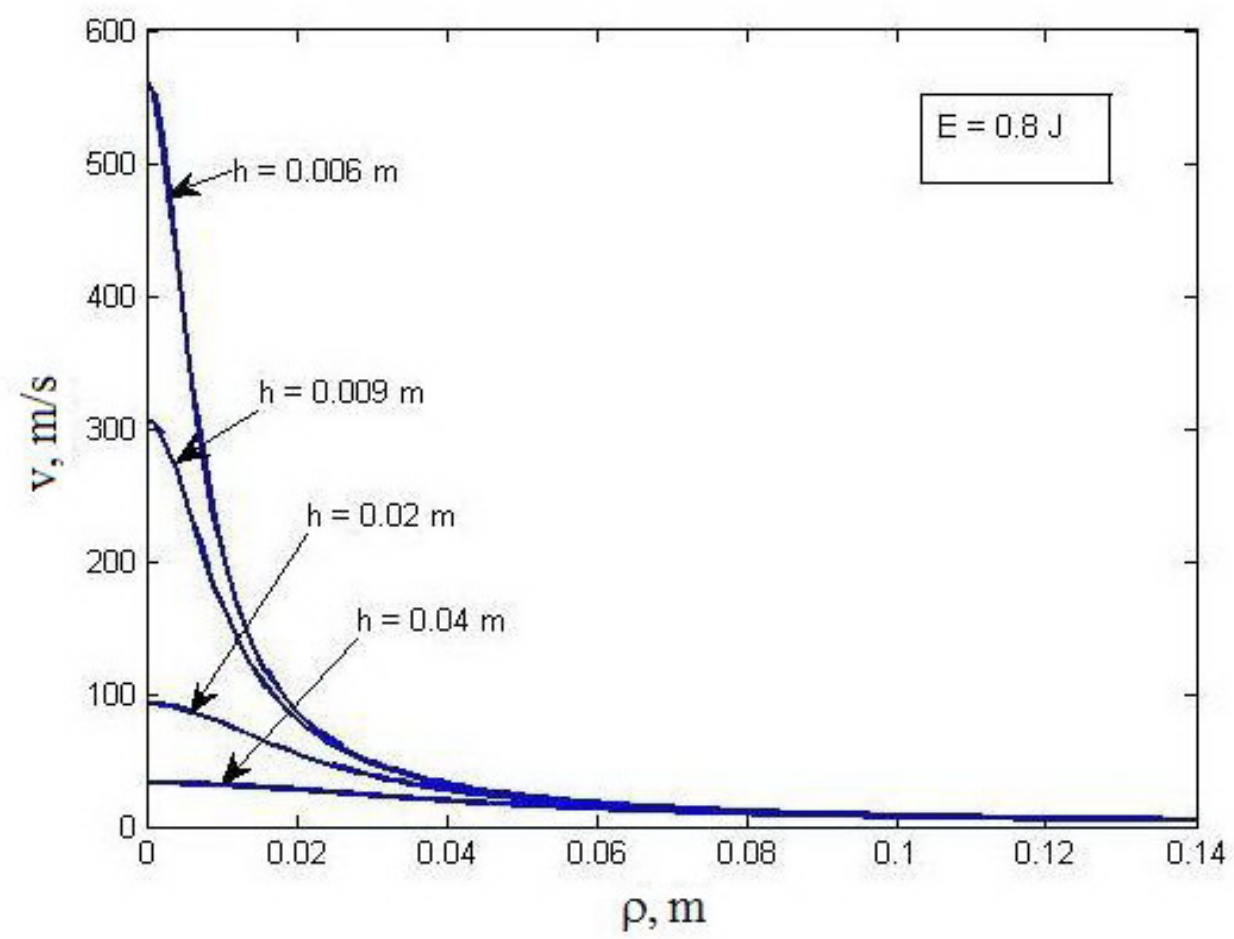

Figure 3: Calculated shock front velocity as a function of height $h$ of the laser spark and distance $\rho$ from the epicenter - (above); calculated pressure pulse in an acoustic wave according to Eqn (8) - (below) 

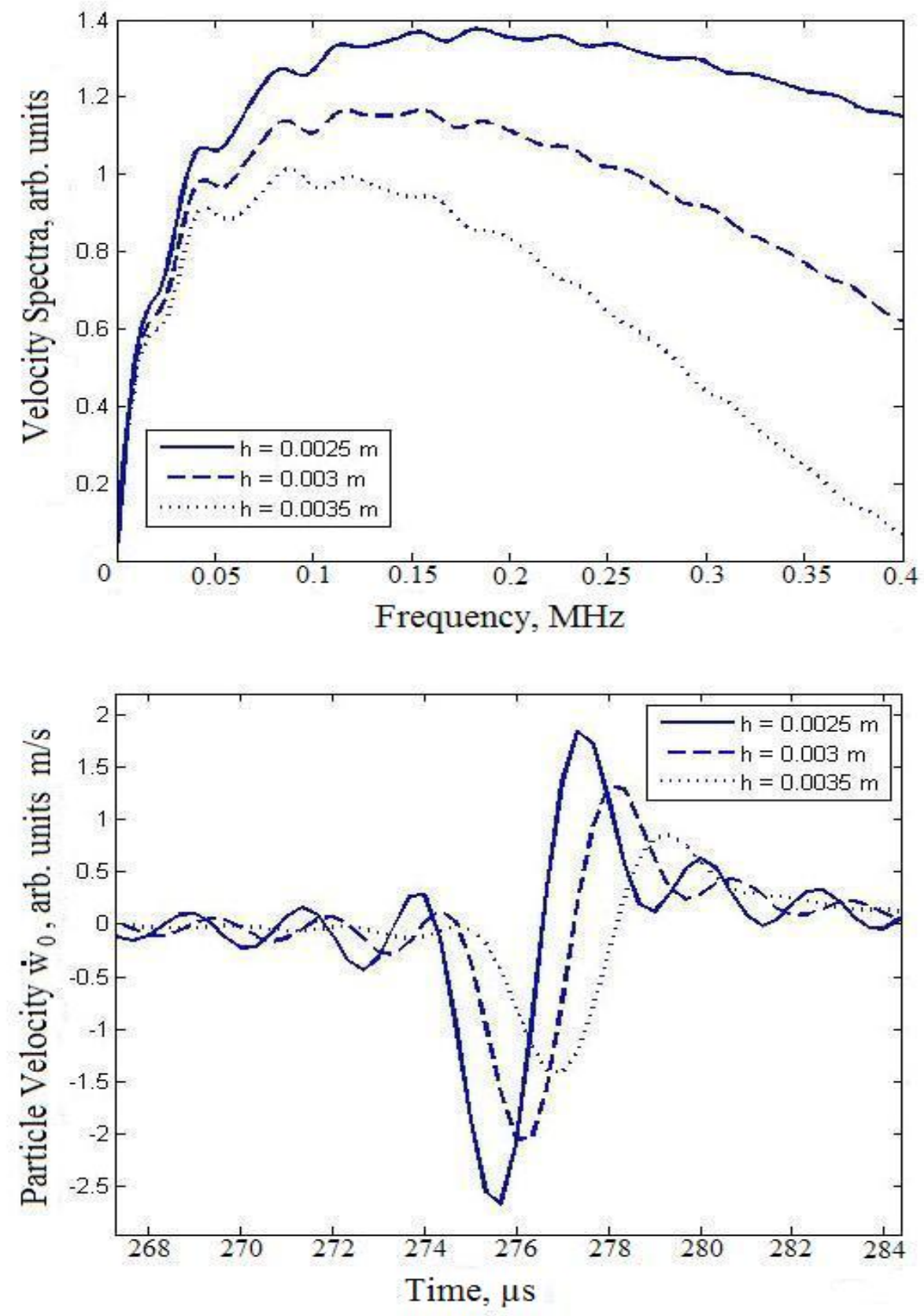

Figure 4: Calculated amplitude spectra (above) and time histories (below) of the Rayleigh-type wave velocity pulses for different heights of the laser-generated spark 

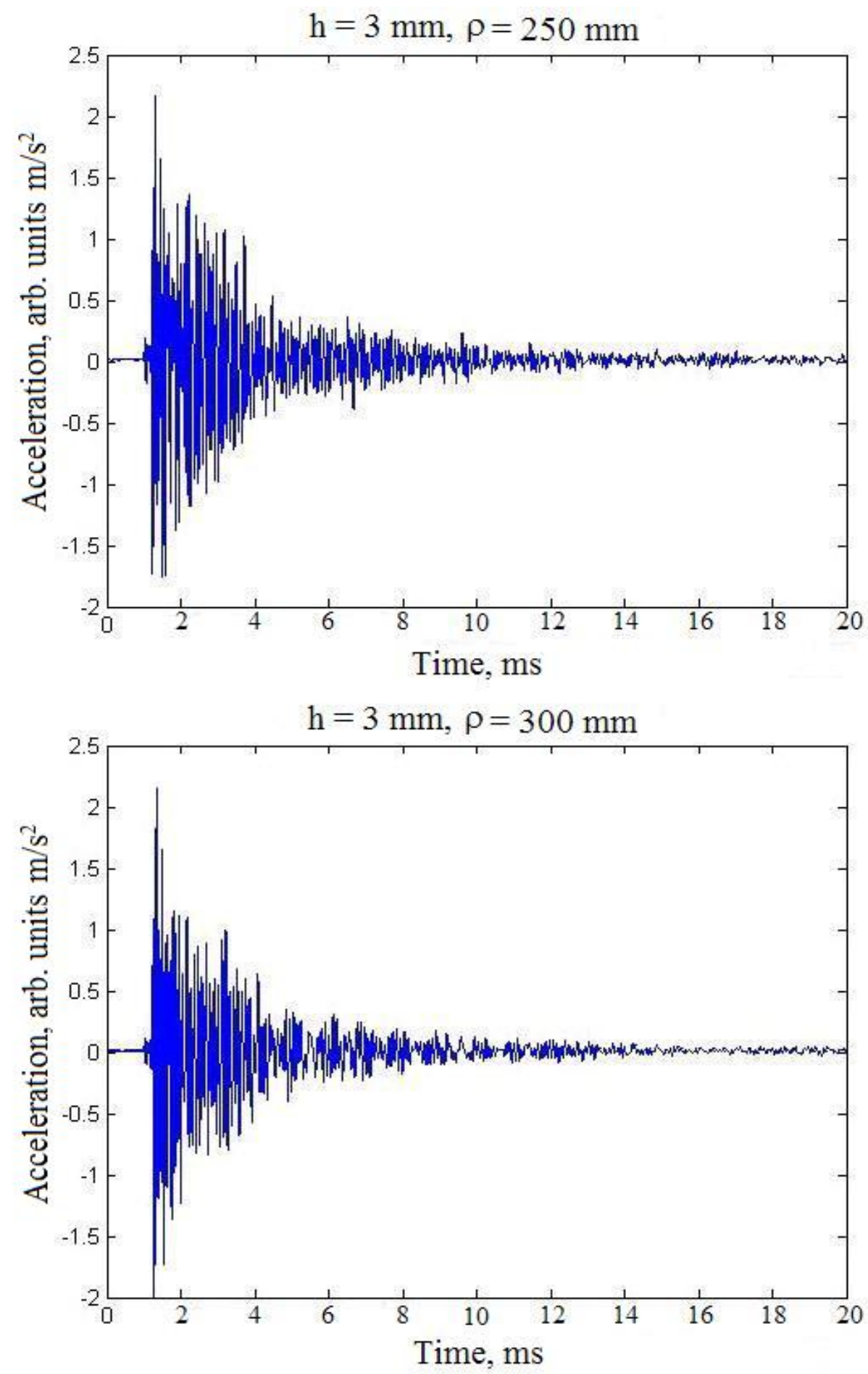

Figure 5: Measured time histories of the acceleration pulses of Rayleigh-type waves at distances of $250 \mathrm{~mm}$ (above) and $300 \mathrm{~mm}$ (below) from the epicenter 

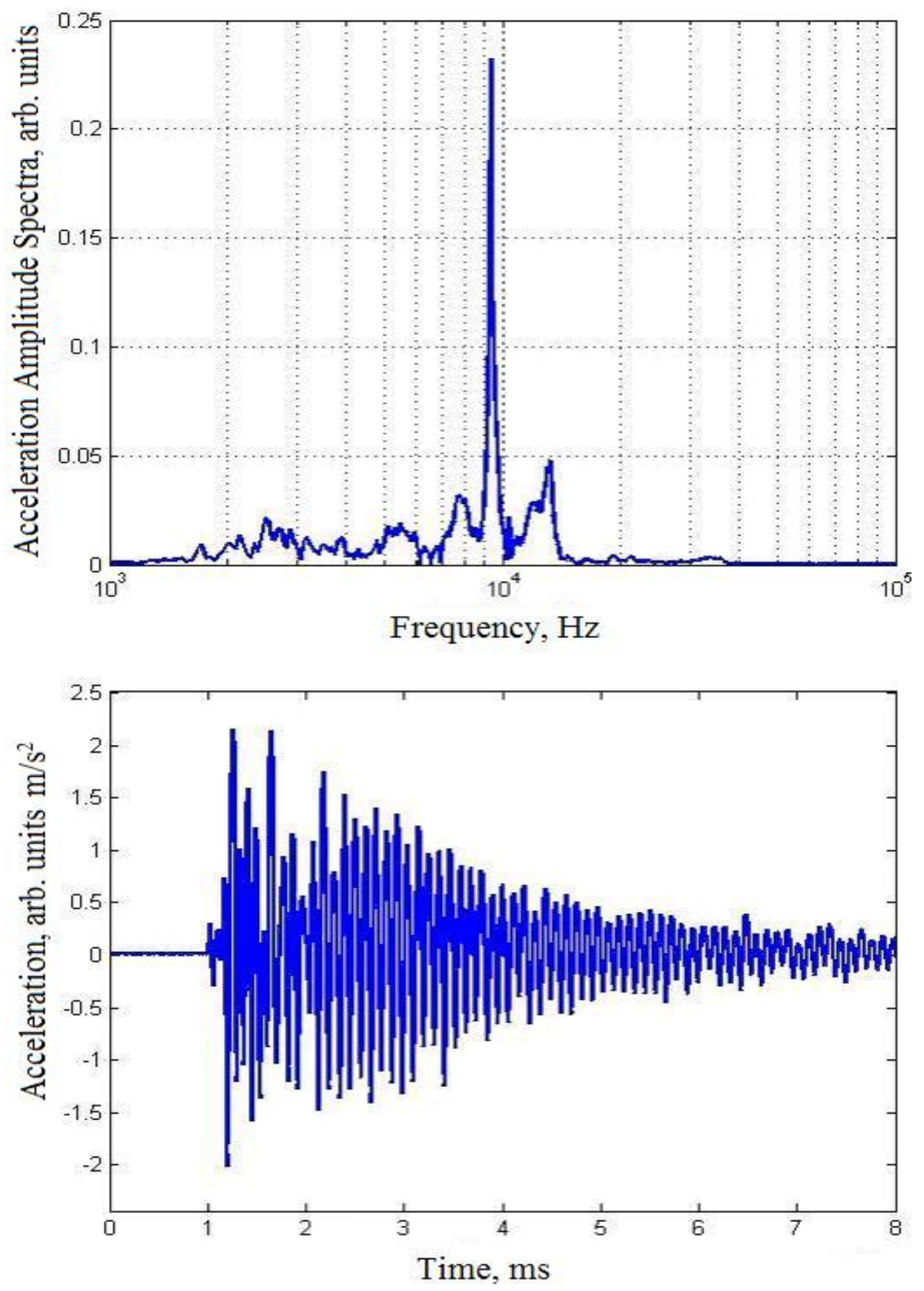

Figure 6: Measured amplitude spectrum (above) and time history (below) of the acceleration pulse of generated Rayleigh-type wave at the distance of 200 $\mathrm{mm}$ from the epicenter 


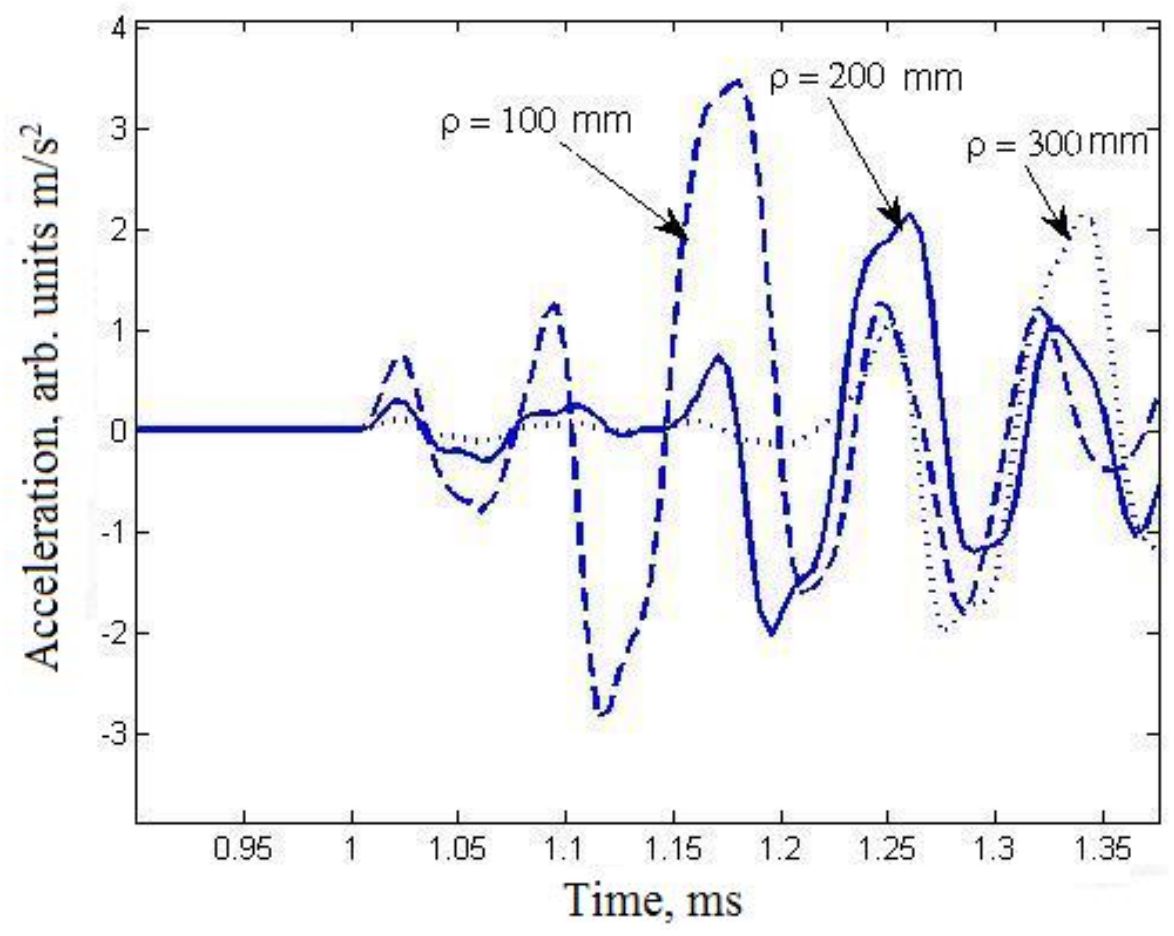

Figure 7: Measured time-delayed acceleration pulses (first oscillations) at the distance of 100, 200 and $300 \mathrm{~mm}$ from the epicenter 


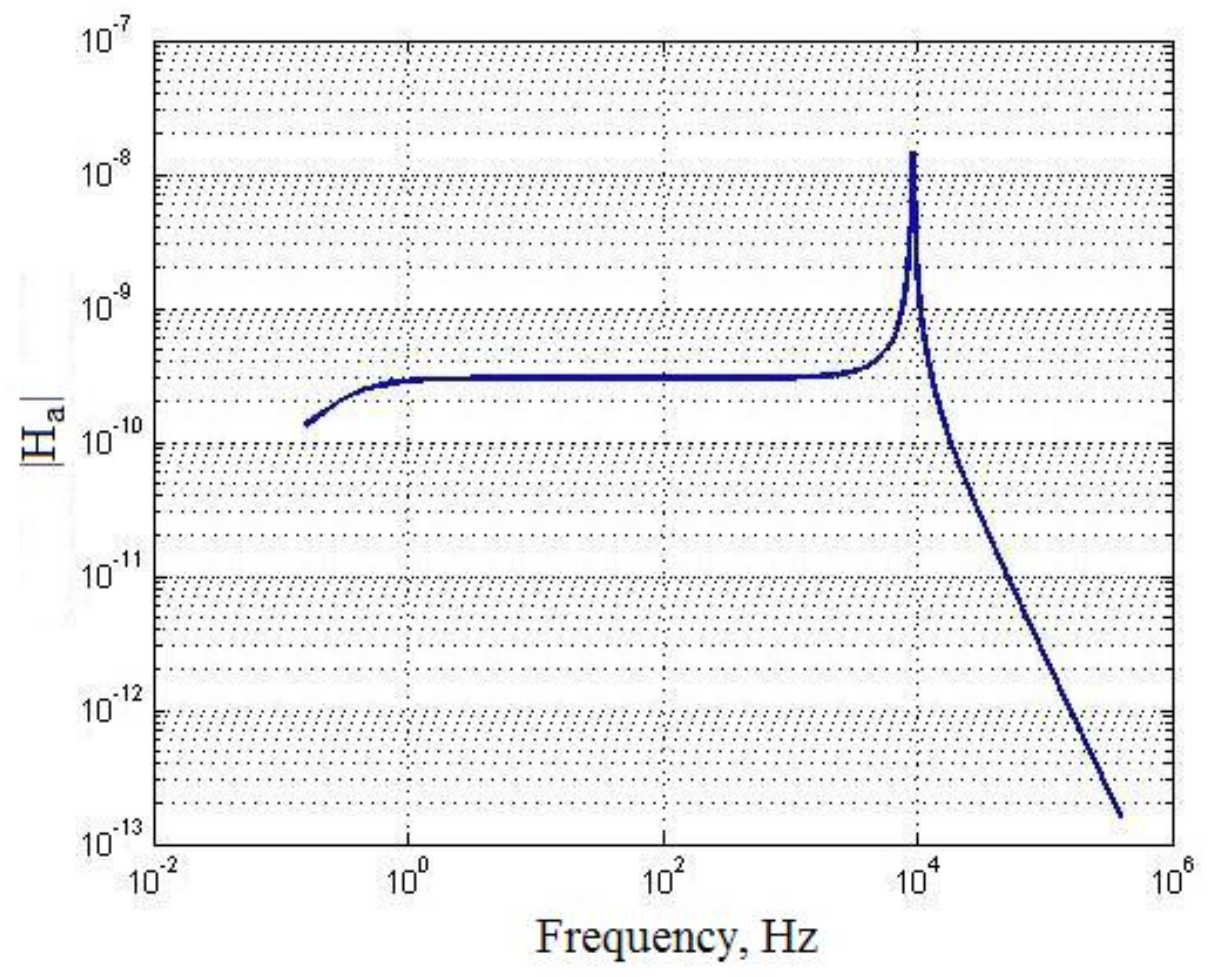

Figure 8: Calculated transfer function $\left|\mathrm{H}_{\mathrm{a}}\right|$ of an accelerometer having a resonant frequency at $9.2 \mathrm{kHz}$ according to Eqn (18) 


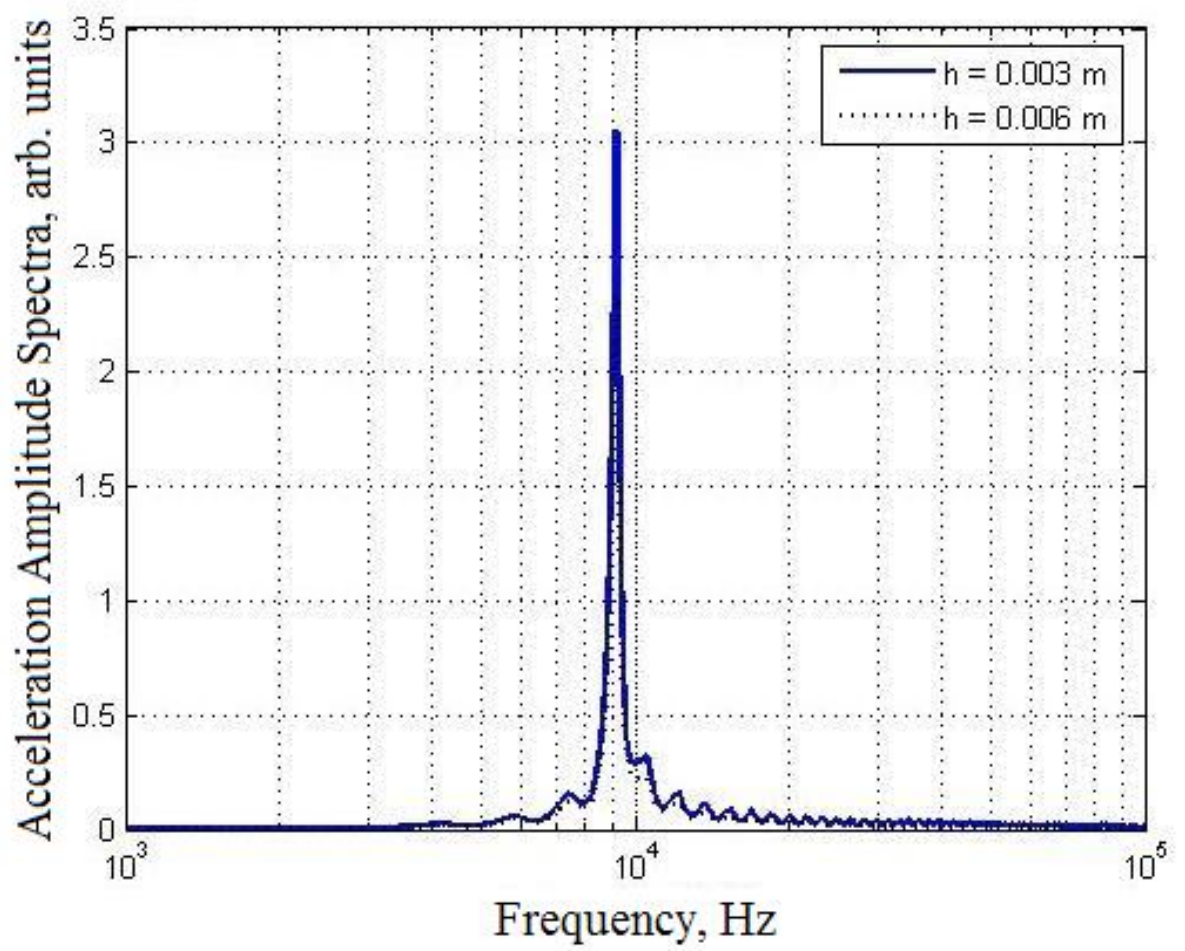

Figure 9: Calculated acceleration amplitude spectrum of a generated Rayleigh-type wave after allowing for the calculated accelerometer FRF; the distance is $200 \mathrm{~mm}$ from the epicenter, and heights are $0.003 \mathrm{~m}$ (solid curve) and 0.006 m (dotted curve) 

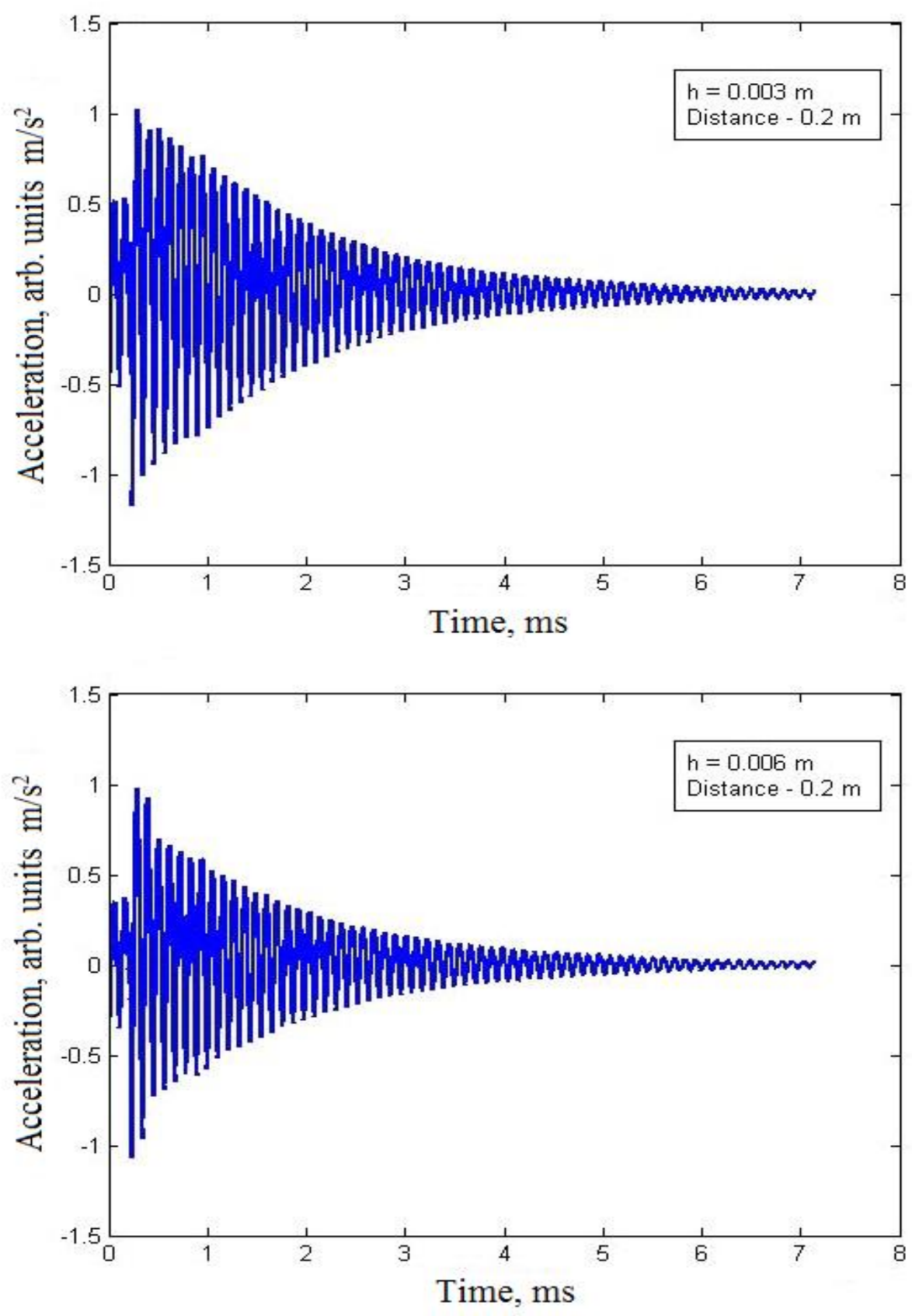

Figure 10: Calculated time histories of the acceleration pulses of generated Rayleightype waves at the distance of $200 \mathrm{~mm}$ from the epicenter predicted after allowing for the calculated accelerometer FRF; heights are $0.003 \mathrm{~m}$ (above) and $0.006 \mathrm{~m}$ (below) 